\title{
Interview with Abel Laureate Robert P. Langlands
}

Robert P. Langlands is the recipient of the 2018 Abel Prize of the Norwegian Academy of Science and Letters. ${ }^{1}$ The following interview originally appeared in the September 2018 issue of the Newsletter of the European Mathematical Society ${ }^{2}$ and is reprinted here with permission of the EMS.

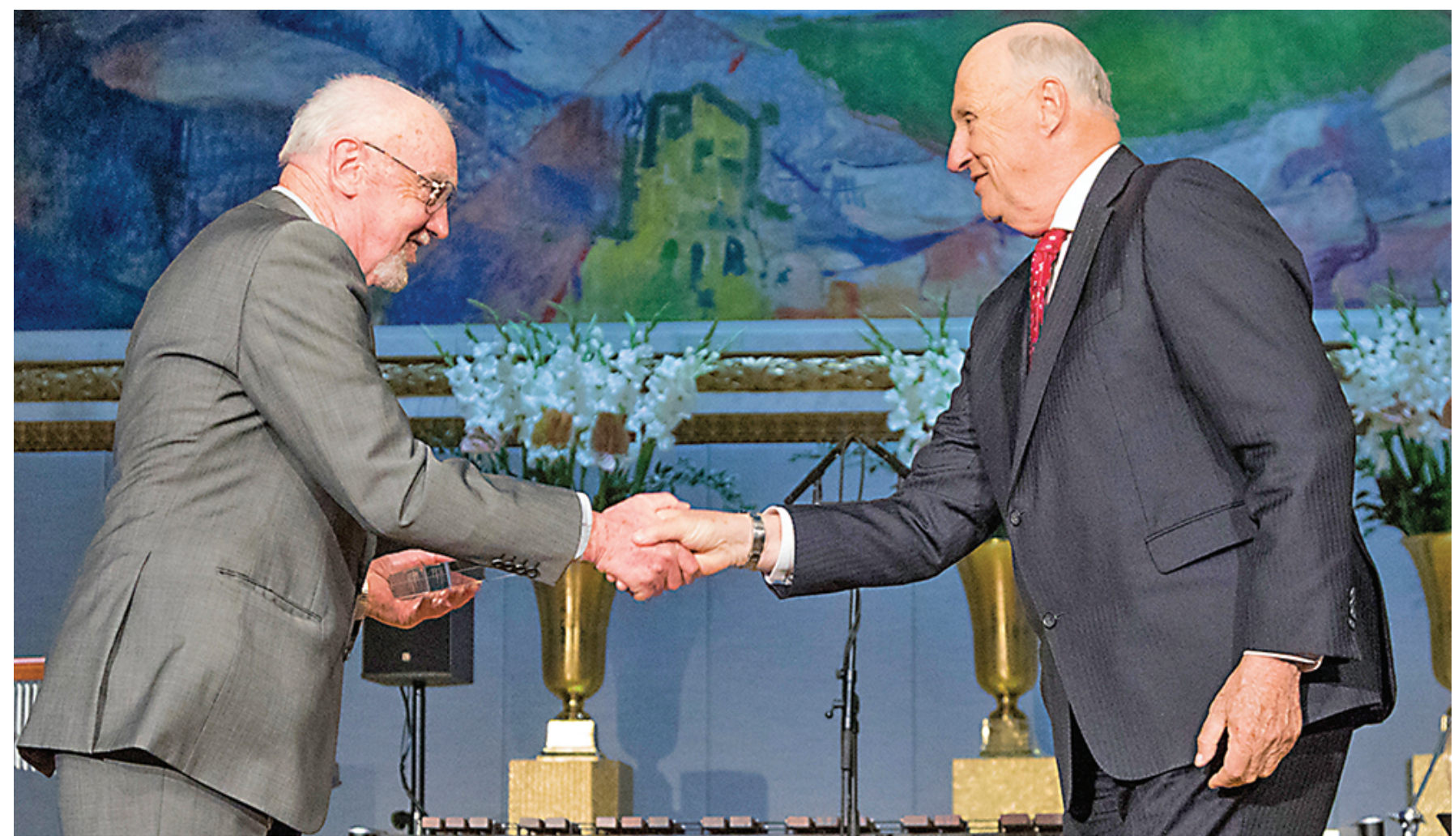

Figure 1. Robert Langlands (left) receives the Abel Prize from H. M. King Harald.

\section{Bjørn Ian Dundas and Christian Skau}

Bjørn Ian Dundas is a professor of mathematics at University of Bergen, Norway. His email address is Bjorn. Dundas@uib.no.

Christian Skau is a professor of mathematics at the Norwegian University of Science and Technology, Trondheim, Norway. His email address is csk amath.ntnu.no.

${ }^{1}$ See the June-July 2018 Notices https: //www . ams . org/journa1s /notices/201806/rnoti-p670.pdf

${ }^{2}$ http: //www.ems-ph.org/journals/news 1etter /pdf/2018-09-109. pdf\#page=21, pp.19-27

For permission to reprint this article, please contact: reprint -permission@ams.org.

DOI: https://dx.doi.org/10.1090/noti 1856
Dundas and Skau: Professor Langlands, firstly we want to congratulate you on being awarded the Abel Prize for 2018. You will receive the prize tomorrow from His Majesty the King of Norway.

We would to like to start by asking you a question about aesthetics and beauty in mathematics. You gave a talk in 2010 at the University of Notre Dame in the US with the intriguing title: Is there beauty in mathematical theories? The audience consisted mainly of philosophers-so non-mathematicians. The question can be expanded upon: Does one have to be a mathematician to appreciate the beauty of the proof of a major theorem or to 
admire the edifice erected by mathematicians over thousands of years? What are your thoughts on this?

Langlands: Well, that's a difficult question. At the level of Euclid, why not? A non-mathematician could appreciate that.

I should say that the article was in a collection of essays on beauty. You will notice that I avoided that word; in the very first line, I said: "Basically, I do not know what beauty is!" I went on to other topics and I discussed the difference between theories and theorems.

I think my response to this is the same today. Beauty is not so clear for me; it is not so clear when you speak about beauty and mathematics at the same time. Mathematics is an attraction. If you want to call it beauty, that's fine. Even if you say you want to compare with the beauty of architecture. I think that architectural beauty is different from mathematical beauty. Unfortunately, as I said, I just avoided the question in the article and, if you forgive me, I will avoid it today.

Dundas and Skau: One other reason we ask this question is that, as you are well aware, Edward Frenkel, who you have worked with and who is going to give one of the Abel Lectures later this week about aspects of the Langlands programme, wrote a best-seller with the title Love and Mathematics and the subtitle The Heart of Hidden Symmetry. The Langlands programme features prominently in that book. He makes a valiant effort to try to explain to the layman what the Langlands programme is all about. We were very intrigued by the preface, where Frenkel writes: "There is a secret world out there, a hidden parallel universe of beauty and elegance, intricately intertwined with ours. It is the world of mathematics. And it is invisible to most of us." You have probably read the book. Do you have any comments?

Langlands: I have skimmed through the book but I have never read it. I am going to say something that is probably not relevant to your question. We are scientists: we ask about, we think about, we listen, at least, to what scientists say, in particular about the history of the Earth, the history of the creatures on it and the history of the Universe. And we even discuss sometimes the beginning of the Universe. Then, the question arises, something that puzzles me although I've seldom thought about it, except perhaps when I am taking a walk-how did it get started at all? It doesn't make any sense. Either something came out of nothing or there always was something. It seems to me that if I were a philosopher or you were a philosopher, we'd have to ask ourselves: how is it that something can be there? It's complicated; it's not irrelevant that the world is very complicated but the enigma is simply the fact that it is there. How did something come out of nothing? You may say with numbers it can happen but beyond that I don't know.

Dundas and Skau: You have your creative moments, where all of a sudden you have a revelation. Hasn't that been a feeling of intense beauty for you?
Langlands: You presumably mean when suddenly things fit together? This is not quite like looking at clouds or looking at the sea, or looking at a child. It is something else; it just works! It works and it didn't work before; it is very pleasant. The theories have to be structural and there has to be some sort of appealing structure in the theory.

But, you know, beauty...women are beautiful, men are beautiful, children are beautiful, dogs are beautiful, forests are beautiful and skies are beautiful; but numbers on the page or diagrams on the page? Beauty is not quite the right word. It is satisfying - it is intellectually satisfying - that things fit together, but beauty? I say it's a pleasure when things fit together.

As I said in the article, I avoided the word beauty because I don't know what it means to say that a mathematical theorem is beautiful. It is elegant, it is great, it is surprising-that I can understand, but beauty?!

Dundas and Skau: But we can at least agree that Frenkel's book was a valiant effort to explain to the layman what beauty in mathematics is and, in particular, that the Langlands programme is a beautiful thing.

Langlands: Well, yes, I would wish that Frenkel were here so I could present my views and he could present his. I have studied Frenkel because he explained the geometric theory but I wasn't interested that much in the beauty. I wanted to read his description of the geometric theory and I got quite a bit from it but I also had the feeling that it wasn't quite right. So, if I wanted to say more, I would want to say it in front of him so he could contradict me.

Dundas and Skau: You have an intriguing background from British Columbia in Canada. As we understand it, at school you had an almost total lack of academic ambition-at least, you say so. Unlike very many other Abel Laureates, mathematics meant nothing to you as a child?

Langlands: Well, except for the fact that I could add, subtract and multiply very quickly. There was an interview in Vancouver-actually, I was in New Jersey but the interviewer, he was in Vancouver-and he asked me a question along those lines and I answered rather frivolously. All the experience I had with mathematics was with arithmetic, apart from elementary school and so on, and I liked to count.

I worked in my father's lumberyard and those were the days when you piled everything on truck by hand and tallied it. And you counted the number of two by fours-is that a concept here? Two by fours: 10 feet, 12 feet, 8 feet, 16 feet....and then you multiply that and add it up with the number of 10 s and multiply by 10 , plus the number of 12 -foot-lengths and multiply by 12 , and so on and so forth and you get the number; convert it to board feet and you know how much it is worth. I would be loading the truck with some elderly carpenter or some elderly farmer from the vicinity. He would have one of these small carpenter pencils and he would very painfully be marking one, two, three, four, five; one, two, three, four, five; and so on. And 
then you would have to add it all up. And me, I was 12, 13 or 14 and I could have told him the answer even before he started. But I waited patiently when he did that.

So, that was my only experience with mathematics except for one or two things, one or two tricks my father used when building window frames to guarantee that the angles are right angles and so on, but that was just a trick, right? The diagonals have to be of equal length if the rectangle is going to be right-angled.

Dundas and Skau: Then, why did you move toward mathematics? Why not languages or other things that you studied?

Langlands: Actually, when I went to university in the almost immediate post-World War II period, it was still regarded as necessary for mathematicians to learn several languages: French, English, Russian or maybe even Italian. Now, that fascinated me. The instruction of French in English-speaking Canada was rather formal; nobody paid too much attention to it. But learning languages rather fascinated me and the fascination has been with me all my life (but that was incidental to mathematics).

Dundas and Skau: Why did you start at university at all?

Langlands: Why did I start...? Here is my conjecture.

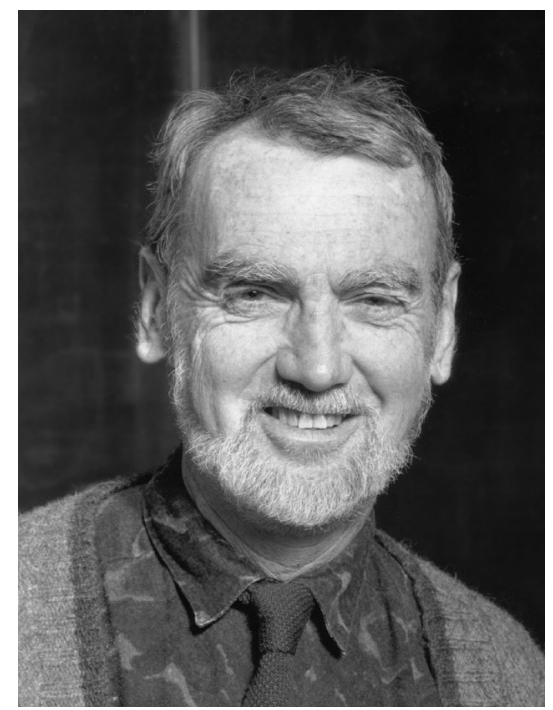

Figure 2. Robert P. Langlands, The Abel Prize Laureate 2018

There are two things (I will come back to the second thing in just a minute). I went to high school. There were children from the neighbourhood and from the surrounding country side, and they tested us. I was indifferent, you know. I didn't pay too much attention but they also used IQ tests and my conjecture has always been that I probably had an unusually high IQquite an unusually high IQ-I don't know. It didn't mean much to me then but that is my conjecture in retrospect. Many of our teachers were just former members of the army in World War II, who were given positions as teachers more as a gratitude for their service in the army. This fellow-he was young, he probably had a university degree and he took an hour of class time to say that I absolutely must go to university. So, I noticed that.

And there was another reason: I had acquired a mild interest in science because I had a book or, rather, my future father-in-law had a book (it was rather a leftist book about eminent scientists; of course, Marx was included, Darwin was included, Einstein was included and so on-various scientists from the 1600s, 1700s, 1800s, etc.) and he gave it to me. He himself had a childhood with basically no education and he learned to read aged about 37, during the Depression when the Labour parties were recruiting unemployed people. So, he learned to read but never very well and I think he never really could write. He always had a good memory so he remembered a number of things and he also had a library and, in particular, he had this book, which was very popular in the pre-war period. So, I began to read this book. My wife-my future wife-had a better idea of what one might do as an adult than I did and she influenced me. And I had this book, where I read about outstanding people like Darwin and so on, and that influenced me a little in the sense that it gave some ideas of what one might do.

And there was also the accident that I always wanted to leave school and hitch-hike across the country, but when I turned 15, which was the legal age when you can stop going to school-I had only one year left-my mother made a great effort and persuaded me to stay another year. During that last year, things were changing for various reasons, e.g. the lecture of that teacher and an introduction to one or two books, so I decided to go to university.

Dundas and Skau: You go on to obtain a Master's thesis at the University of British Columbia, you marry and then you go to Yale and start a PhD in mathematics. It is quite a journey that you were on there. How did you choose the thesis topic for your PhD at Yale?

Langlands: First of all, Hille had this book-you may know it-on semi-groups and I was an avid reader of that book, and I took a course from Felix Browder on differential equations. You may not know but Felix Browder was an abysmal lecturer and so you had to spend about two or three hours after each lecture sorting things out. He knew what he was talking about but it took him a long time to get to the point or to remember this or that detail of a proof. I went home and I wrote out everything he had talked about.

So, I had this background in partial differential equations from his course and I had read all of Hille's book on semi-groups and I just put the two together. I really liked to think about these things.

Dundas and Skau: In other words, you found your own PhD topic?

Langlands: Yes, I found my own PhD topic.

Dundas and Skau: But from there on, after your thesis, we have what we like to think of as a journey toward a discovery. Your work on Eisenstein series and your study of the theory of Harish-Chandra are crucial ingredients here. Would you care to explain to us what the background was that led to the Langlands programme?

Langlands: There was a Hungarian fellow, S. Gaal, who had immigrated to the US after the difficulties in Hungary and that was in the middle of the 1950s. The Norwegian mathematician Atle Selberg was a member of the Institute of Advanced Study (IAS) in Princeton. Selberg's wife was 
Romanian and spoke Hungarian and I think Gaal (he and his wife and maybe their children too) was invited to the IAS by Selberg. He had come to the US sponsored more or less by Selberg and he was giving a graduate course at Yale, where he talked about Selberg's paper, basically at the time of Selberg's second so-called "Indian paper," a Tata publication from 1960. Selberg didn't write that many papers at the time but I think there were two and Gaal talked about that. Also, I have to mention that there was an important seminar on convexity in the theory of functions of several complex variables.

So, you hear about Selberg and you hear about Eisenstein series, and this theory about convexity, and then you want to prove things and you move more or less instantly to an analytic continuation of Eisenstein series in several variables. So, I had already thought about that but I thought about them in a rather restricted context-no algebraic numbers, for example.

And then I got a position at Princeton University, not because of anything I had done about Eisenstein series but because of my work on one-parameter semi-groups. So, I gave a lecture in one seminar; Bochner didn't run it but he kept an eye on it. I think he was impressed simply because I was talking about something that wasn't in my thesis. I talked about this work with Eisenstein series and I think he was impressed by me. Now, Bochner's family was from Berlin. He wasn't born there but he lived there as a child. He went to German universities and he had connections with Emmy Noether and Hasse, for example. So, he took an interest in anything that had to do with algebraic number theory and he encouraged me to think about Eisenstein theory in a more general context, not just for groups over rational numbers but also for groups over algebraic number fields.

Dundas and Skau: So Bochner was almost like a mentor for you for a while?

Langlands: Not a mentor but he was like a foster father, if you like. He encouraged me-more than an encouragement; he pushed me. Bochner encouraged me to work over algebraic number fields rather than just over the rational number field. Algebraic number fields I basically learned from Hecke and I read papers by Carl Ludwig Siegel (because there are ways to handle analytic continuation of series, which you can take from Siegel's papers). I started to read a little in the literature of these two, Hecke and Siegel, and I wrote about Eisenstein series basically using their very classical methods.

In any case, one year-just about a week before the classes were to start-I was going to give a course in class field theory. Emil Artin had been in Princeton and was the expert on class field theory; he had gone back to Germany in 1958 and there were one or two disappointed students who had come to Princeton to learn a little bit of class field theory. There was no real information on class field theory to be obtained from the courses offered. I had attended a seminar that was arranged by these disappointed students but it wasn't such a good seminar, so I was quite ignorant. But Bochner said: "You are to give a course in class field theory." And I said: "How can I do it? I don't know anything about it and there is only one week left." But he insisted so I gave a course on class field theory from Chevalley's paper, which is the more modern view, and I got through it. There were three or four students, who said they learned something from it.

So, with that, I began to think about the fact that there was no non-abelian class field theory yet. Some people, like Artin, didn't expect there to be any. So, I was just aware of it, that's all. We are now in August of 1963 or something.

Dundas and Skau: You already had a position at Princeton University at the time?

Langlands: I had a comfortable position at the university and I went up the ladder reasonably rapidly. I think by 1967, I was an associate professor or something like that. Thanks to Selberg, I was at the IAS for a year, and I was at Berkeley, California, for a year. So, I was away two times.

Dundas and Skau: And all this while you were contemplating the trace formula, is that correct?

Langlands: Well, let me go back. I have forgotten something. I was concerned with the trace formula and I wanted to apply it. The obvious thing you want the trace formula for is to calculate the dimension of the space of automorphic forms; that is the simplest thing. So I wanted to do it. And, so, you plug in a matrix coefficient-as I understood it; it doesn't look like a matrix coefficient_of an infinite dimensional representation into the trace formula and you calculate.

I didn't quite know what to do with this and then I spoke to David Lowdenslager-he died very young — and he said: "Well, people are saying that this is really something you can find in Harish-Chandra." So, I started to read Harish-Chandra and what I observed very quickly, because of reading Harish-Chandra, was that the integral that was appearing in the trace formula was an orbital integral of a matrix coefficient. And that orbital integral of a matrix coefficient, we know from representations of finite groups, is a character and, basically, you learn from Harish-Chandra's paper that this is indeed the case. So, that meant that I had to start to read Harish-Chandra-as I did.

And once you start to read Harish-Chandra, of course, it goes on and on; but that was the crucial stage: this observation of Lowdenslager that people were beginning to think that Harish-Chandra was relevant. So, there we are, we have it all. And then I began to think about these things, slowly; and sometimes it worked out, and sometimes it didn't. I could actually apply the trace formula successfully.

In 1962, Gelfand gave a talk at the ICM in Stockholm and a year later his talk was circulating. Now, Gelfand gave his views of the matter. The point was that he introduced the notion of cusp forms explicitly. The cusp form is a critical notion and it is a notion that I think appears in rather 
obscure papers by Harish-Chandra and Godement. But it is hard; you have to look for it. But with Gelfand it was clear why that was so fundamental. Now, an incidental question: I don't think Selberg ever really grasped the notion of a cusp form. Selberg, of course, didn't read other people's papers and I don't think he ever grasped the notion of a cusp form. I think that was an obstacle that he never overcame.

But as soon as you read Gelfand, you can do it-you can prove the general theory about Eisenstein series. You have to know something. In other words, you have to be someone who knows something about unbounded operators on Hilbert spaces. You have to be someone with this background or it doesn't mean anything to you. But if you had that background then you saw immediately what was to be done: take what Selberg had done in rank one to the general case.

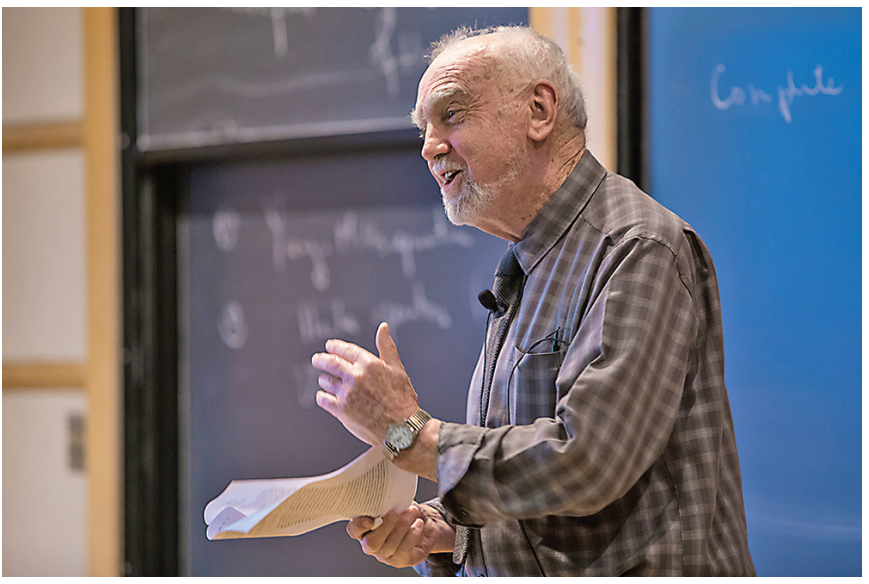

Figure 3. Robert P. Langlands giving a lecture.

Let me go back a little. I only talked mathematics with Selberg once in my life. That was in 1961, before I came to the Institute (IAS). It was at Bochner's instigation, I am sure. Selberg invited me over and he explained to me the proof of the analytic continuation in rank one. Now, of course, the proof of the analytic continuation in rank one is like Hermann Weyl's theory on differential equations of second-order on a half-line. I had read Coddington and Levinson's book Theory of Ordinary Differential Equations not too long before, so I could just sit there and listen to Selberg-listen to the kind of things he knew very well—and he explained it to me. Whether he regretted that afterwards I cannot say but he explained to me how it works in rank one.

Dundas and Skau: Were you impressed by his presentation?

Langlands: I had never spoken mathematics with a mathematician on that level before in my life. I had really never spoken mathematics with Bochner and he is the one that came closest.

Dundas and Skau: Even so, you didn't have further conversations with Selberg afterwards.

Langlands: No, he wasn't a talkative man. Well, I did have occasional conversations because then I was still continuing to try to prove the fundamental analytic contin- uation of Eisenstein series. I approached him when I had done it in this or that case and he'd say: "Well, we don't care about this or that case. We want to do the general theory." So he didn't listen to me. While we were colleagues and our offices were basically side by side, we'd say hello but that's about it.

Dundas and Skau: So you spent many years together in virtually adjacent offices at the IAS and you never really talked mathematics?

Langlands: No. Selberg, you must know, didn't speak with very many people about mathematics. He spoke with one or two, I think, but not many. I am not sure how much he thought about mathematics in his later years. I just don't know.

Dundas and Skau: But even so, your work on Eisenstein series really had some consequences in hindsight, didn't it?

Langlands: Yes, it was critical in hindsight, right? So, that took me about a whole year and I think I was exhausted after that-it was one of the cases where you think you have it and then it slips away. There was, for example, an induction proof. In induction proofs, you have to know what to assume: if you assume too much it is not true and if you assume too little it doesn't work.

In fact, there was a problem; things were happening that I didn't recognise. In other words, it could be a second-order pole where you naturally assume that there is only a first-order pole. And it took me a long time to reach that stage. Specifically, it is the exceptional group $G_{2}$ of the Cartan classification. You think this is going to work; and you try and it doesn't work-it doesn't work in general. Then you think about what and where it can really go wrong and it turns out that it only goes wrong for $G_{2}$. Then you make a calculation with $G_{2}$ and what do you see? You see this second-order pole or a new kind of first-order pole and that changes the game: you have a different kind of automorphic form.

It eventually worked; it was an exhausting year but it did eventually work.

Dundas and Skau: And in the Autumn of 1964, you went to Berkeley, is that correct?

Langlands: And then I went to Berkeley, pretty much exhausted by that particular adventure.

Dundas and Skau: Were you really so exhausted that you thought about quitting mathematics?

Langlands: Well, look, quitting mathematics is a rather strong statement. But I did decide to spend a year in Berkeley and got some things done in retrospect. I did more than I thought I had done. I was too demanding, you know. When you are younger, you are a little more demanding than when you are older. So, the next year I was really trying, I think, to do something with class field theory and I didn't see anything. I had a whole year where I don't feel I got anything done. In retrospect, in Berkeley, I did something but the year afterwards I didn't at first do anything and I was growing discouraged. 
So, I decided on a little bit of foreign adventure. I pretty much decided that the time was right. I should just go away and maybe think of doing something else. I had a Turkish friend and he explained to me the possibility of going to Turkey. So, I decided to do that and, once I had decided to go to Turkey, there were various things to do; I wanted to learn some Turkish and then I went back to studying Russian. I had a very nice teacher. But I still had a little time to spare and I didn't know quite what to do and I began to calculate the constant terms of Eisenstein series.

Dundas and Skau: Just for the fun of it?

Langlands: Just for having something to do. And so I calculated them. I just calculated it for various groups and then I noticed that it was basically always of the form $f(x) / f(x+1)$ or something like that. But if you could continue the Eisenstein series you could continue the constant term and instead of $f(x) / f(x+1)$, you could continue $f(x)$. And these things are Euler products, so you have new Euler products. Of course, analytic number theorists just love Euler products. So you had it! You had something brand new: they had an analytic continuation and a functional equation. And you could basically do it for a lot of groups.

Dundas and Skau: You could even do it for reductive groups?

Langlands: You basically did it for split groups, i.e. those reductive groups with a split maximal torus, and then you have the classification. So, you had a whole bunch and, if you looked at them, you could see that somehow they were related to representations of Eisenstein series associated to parabolic groups of rank one. And they were somehow related to a representation; you have a parabolic group and you take the reductive subgroup-it is of rank one and you throw away the rank one part so you basically have some kind of $L$-function associated to the automorphic form on this reductive subgroup.

All right, so you have Euler products that are attached to a representation of a group. Euler products are Dirichlet series that number theorists love-and that is what you want. You have a large list of groups. And that already suggests something. You can formulate this-you can see somehow where this is coming from. You can see how to formulate it as a representation associated to an automorphic form and a particular representation of what I call the $L$-group, for $L$-series.

And there you are: you start to make a guess and you have this in general! For a particular reductive group, you have an Euler product with an analytic continuation, associated to a representation. But you think it works in general. So, once you have that-once you have something that might work in general-you have to think of how you are going to prove it in general.

Dundas and Skau: This must have been extremely exciting?

Langlands: Well, it was!

Dundas and Skau: Incidentally, did you continue with your classes in Russian or Turkish?
Langlands: I gave up both, even the Russian class where the teacher was this sweet woman; I think she liked me since I was an industrious student. She was very angry and wouldn't talk to me.

Dundas and Skau: Is it fair to say, then, that your discovery comes out of...well, you were extremely exhausted, you let your shoulders down, you play, you have some evidence and you make a major discovery?

Langlands: I think that's an apt description.

Dundas and Skau: When did you have this epiphany, if you like, where you saw the connection with the Artin conjecture about the analytic continuation to the whole complex plane of the Artin L-functions?

Langlands: It was during the Christmas vacation of 1966. Although I have forgotten the date the idea came to me, I still have a vivid recollection of the place. In the old Fine Hall at Princeton University, there was a small seminar room on the ground floor directly to the east of the entrance. The building itself, I recall, was of a Gothic style with leaded casement windows. I was looking through them into the ivy and the pines and across to the fence surrounding the gardens of the President's residency when I realised that the conjecture I was in the course of formulating implied, on taking $G=\{1\}$, the Artin conjecture. It was one of the major moments in my mathematical career. ${ }^{3}$

Dundas and Skau: Was this a so-called Poincaré moment for you? You know the story about Poincaré getting on a bus when all of a sudden he saw the solution to a problem he had been thinking about for months and then put aside.

Langlands: Except that somehow I was not searching. I had no idea I would stumble across a non-abelian class field theory.

Dundas and Skau: And this was right before you sent the 17-page, handwritten letter to André Weil outlining your theory?

Langlands: Yes. The letter to André Weil is somehow an accident. The point is, I went to a lecture by Chern. Weil went to the same lecture and we both arrived early. I knew him but not particularly well. We both arrived early and the door was closed so we couldn't go in. So, he was standing there in front of the door and I was standing there in front of the door. He wasn't saying anything so I thought I should say something. I started to talk about this business. And then he didn't understand anything, of course, and he probably behaved as you'd behave under those circumstances; I was this fellow talking to him and I just assumed he would walk away but he said "write me a letter." I wrote him a letter. He never read the letter so far as I know.

Dundas and Skau: He had your letter typed and distributed, didn't he?

Langlands: Yes, that's right.

\footnotetext{
${ }^{3}$ Langlands (2005): The genesis and gestation of functoriality. publications.ias.edu/sites/defau $1 \mathrm{t} / \mathrm{fi} 1 \mathrm{es}$ /TheGenesis.pdf
} 
Dundas and Skau: This is not the only moment you describe where you are making a discovery while not sitting behind your desk and working. On another occasion, you tell of how you are walking from here to there and suddenly you see something. Is that a pattern of yours? Is that how you find things?

Langlands: I have certainly seen these things very seldom in my life so I don't think one can speak about a pattern.

Dundas and Skau: Perhaps it is time that you actually tell us about what the Langlands programme is all about? Just in broad brush strokes.

Langlands: Okay, we sort of know what the quadratic reciprocity law is, right? There, two things that appear to be quite different are the same. Now, we also know that, after Weil, we can define zeta-functions (or $L$-functions would probably be better). You can define them over finite fields and you can also define them if you have a global field and you take the product of the ones over finite fields and you get some kind of an $L$-function associated to a variety or even, if you like, to a particular degree of the cohomology of that variety.

A basic problem in arithmetic for any kind of estimation of the number of solutions of Diophantine equations is reflected in the $L$-functions that you can formally associate-and you are in the half-plane - to the cohomology of the given degree of any kind of curve over a number field. They are there.

Presumably, if you can deal with these then you can, somehow or other, do more things about the estimation of the number of solutions and the nature of solutions. I think no one has a clear idea about this, except in very specific cases, i.e. what you can do with the knowledge of these global $L$-functions. But they are there, and you want to prove that they have analytic continuation. The only reasonable way, on the basis of evidence, is that they will be equal to automorphic $L$-functions.

Now, from the point of view of the variety and the cohomology of the variety, you have the Grothendieck formula. I don't know to what extent he actually had a complete theory-I don't think he had-but he had the notion of a motive, and a motive has certain multiplicative properties. So, you had a whole family of functions that behaved in a natural functorial manner. And you wanted to prove that they could be analytically continued. But he managed to associate a group; in other words, these motives were associated to representations of a group, whose nature had to be established. On the other hand, the group is there: you may never know its nature but you should be able to find out its relations to other groups.

Now, on the other hand, what you would like, normally, in order to establish the analytic properties of these things that are defined algebraically/geometrically is to associate them to something that is defined analytically because automorphic $L$-functions basically have analytic continuations. There are some questions about it, right, because you can do it if they are associated to $\mathrm{GL}_{n}$ and the standard representations of $\mathrm{GL}_{n}$ (that is the theorem by Jacquet and Godement from 1972). But, in the end, you need to do two things that are more or less mixed, namely, for an automorphic form associated with a general group, you need to show that that automorphic form really sits on $\mathrm{GL}_{n}$; you push it toward $\mathrm{GL}_{n}$ and then you define the $L$-function. So, it is not just an automorphic form but it is an automorphic form that can be pushed toward $\mathrm{GL}_{n}$.

Now, that will make you think that somehow an automorphic form is associated to a representation of a group, which has to be defined. In other words, there is a structure in the connection of all automorphic forms. You can pass it from one associated with $G$. (It is not true that if you associate it with $G$, you can pass it to another group $G^{\prime}$ if $G$ goes to $G^{\prime}$.)

This is the so-called $L$-group and you have to push it forward. If you have this motion and you can push, you could say you have the automorphic form here equal to one over there, and so the $L$-function is the same.

If the one you take over here is $\mathrm{GL}_{n}$ then you know, by Jacquet-Godement, that you can handle it. So, if you have a way of passing-whenever you have the form on one group-to other groups in the appropriate formalism then you can handle analytic continuation.

Dundas and Skau: This is what you call functoriality?

Langlands: Yes, this passing like that. So, this means that you can describe it by representations of a group. So, this is the same thing; something similar is happening over on the algebraic/geometric side. And there it is another group; it is the group defined in a similar way and that is the group of Grothendieck and its motive. And when you have the two, you can do all the analytic continuation you want and what you get is, of course, something for your great-grandchildren to discover.

Dundas and Skau: It seems like a very naive question, and it is, but let's ask it anyway. Why is it so crucial to analytically or meromorphically continue the L-functions?

Langlands: Why is that so crucial? That is a good question. Why is it so crucial to know anything about the zeta-function? Where do you go? In other words, you go for an estimate of the number of solutions and things like that. What do you do with the information you have about the zeta-function? And what would you do if you have all the possible information? Do you have an answer?

Dundas and Skau: No, we don't.

Langlands: Neither do I but I think, in both cases, it is that we haven't worked with it in the right area.

Dundas and Skau: Of course, we know that the classical zeta-function tells us something about prime numbers and their distribution. And Dirichlet's L-functions tell us something about prime numbers in arithmetic progressions.

Langlands: So, you get that kind of information but...

It is clear that it is what people are hoping for. But you can ask: why do they want it? Only God knows. So, you're 
pushed by preconceptions and you're trapped in the way you think mathematics should work.

Dundas and Skau: In 2009, the so-called Fundamental Lemma, conjectured by you in 1983, was proved by Ngô. He was awarded the Fields Medal in 2010 for this. Time Magazine selected Ngô's proof as one of the Top Ten Scientific Discoveries of 2009.

Langlands: You can cancel your subscription to Time Magazine!

Dundas and Skau: In a joint paper from 2010 titled "Formule des traces et fonctorialité," the authors being you, Ngô and Frenkel, the very first sentence-translated into English-reads: "One of us, Langlands, encouraged by the work of one of us, Ngô, on the Fundamental Lemma, whose lack of proof during more than two decades was an obstacle for a number of reasons for making serious progress on the analytic theory of automorphic forms, has sketched a programme to establish functoriality-one of the two principal objects of this theory." Any comments?

Langlands: The Fundamental Lemma is needed to deal with a specific kind of technical question. Let's see if I can make it clear. This is not a good example but I'll try to explain something. Say you have something such as the group $\mathrm{SL}_{n}$ and you have $\mathrm{SU}_{n}$. You know by Weyl's theory about the representations of $S U_{n}$. Those are basically the standard finite dimensional representations of this group. Now, look at the $\mathrm{SL}_{n}$ situation; $\mathrm{SL}_{n}$ has more representations than $\mathrm{SU}_{n}$. $\mathrm{SL}_{n}$ is a non-compact group; it has a lot of representations. But, in particular, it has some things that are very much like those of $\mathrm{SU}_{n}$; the characters are basically the same. For example, you know the characters of $\mathrm{SU}_{2}$.

Now, lets go to $\mathrm{SL}_{2}$. By Harish-Chandra's theory-actually, $\mathrm{SL}_{2}$ is prior to Harish-Chandra-you have corresponding representations. In this whole theory of representations of semi-simple groups or reductive groups, and therefore the theory of automorphic forms, and therefore the whole theory, what happens for $\mathrm{SL}_{2}$, for example-those things where there is only one? I mean, you know $\mathrm{SU}_{2}$, where there is only one representation in each dimension. Each one has basically something corresponding for $\mathrm{SL}_{2}$, the so-called discrete series, and at each end, it has two. It is just this one place where this unitary group becomes two for $\mathrm{SL}_{2}$.

These two are, for all practical purposes, the same; they're just two pieces. Now, considering the Fundamental Lemma and what you have to do if you are worrying about the trace formula: you want some part that is really useful for, say, $\mathrm{SL}_{2}$ and that's the part where you put these two together so they look like $\mathrm{SU}_{2}$. Then, there is a supplementary part where you have to take into account the fact that they don't occur with the same multiplicity so you have this extra stuff. So, if you want to handle the trace formula, you have to see what you want to compare. You have to say that $\mathrm{SU}_{2}$ is more or less like $\mathrm{SL}_{2}$. So, you can compare the trace formula of the two but the extra bit over here is causing you trouble. And the reason is that somehow the one representation here breaks up into two representations there, and some of it doesn't have much to do with things and it is just there. You just take the difference of the characters rather than the sum.

If you are going to use the trace formula, you have to understand the part you don't really want. And there is some mysterious endoscopy. What is the so-called Fundamental Lemma? It is a fundamental lemma in the context of the specialised theory that was introduced for this special feature where things that should be the same could sometimes differ. What you do is that you treat them all as if they were the same and put them together and then you take the difference. You have to treat those differences separately so they look like something coming from the torus itself, the circle group that is sitting in there. So, it is a technical necessity; if you want to compare the representations of two groups you use the trace formula, but this stuff, this extra stuff, you have to get it out, put it aside and treat it separately, so you can compare what is left. And then what matters is just to understand what you can compare on its own. That means that you have to understand the differences-you have to look at just the circle group, which is all that matters, and for that you need the Fundamental Lemma, and that's all. The Fundamental Lemma is the fundamental lemma for these technical properties. It's a whole theory for this; it's rather complex but it takes care of that.

Dundas and Skau: Functoriality is the most important part of the Langlands programme. And to make progress on functoriality you have said you think that the crucial tool is going to be the Selberg-Arthur trace formula. Why is the trace formula going to be so important?

Langlands: Well, what do you want to show? You want to show that you can transfer everything to $\mathrm{GL}_{n}$, basically. Let's put this somewhat differently. You want to show that you can move automorphic forms from one group to another. This is something you want to use the trace formula for: you compare the two trace formulae, right?

You want to move things from the group $G$ to the group $G^{\prime}$. You want to be able, in particular, to handle the $L$-function, so you want to be able to move to $\mathrm{GL}_{n}$. These things work at the level of the L-group but let's just work with $\mathrm{GL}_{n^{\prime}}$ so we don't have to worry about that. So, how are we going to do it?

You say here is this group; every time I have a homomorphism of the group-really of the L-group-from one to the other then I have a transfer representation. This means that every representation is obtained by transfer; it is a natural transfer. You can see this if you see the distribution of conjugacy classes. So, what would you do? There is, so to speak, a smallest place, a smallest group where it sits and then it propagates to the other groups.

For example, say, you have one group $G$ that you want to understand. So, you say here is the smaller group, so it has to be the contribution of those things that sort of sit inside the bigger groups in that smaller group, so one-away, one-away you do it all along, moving from the larger to the 


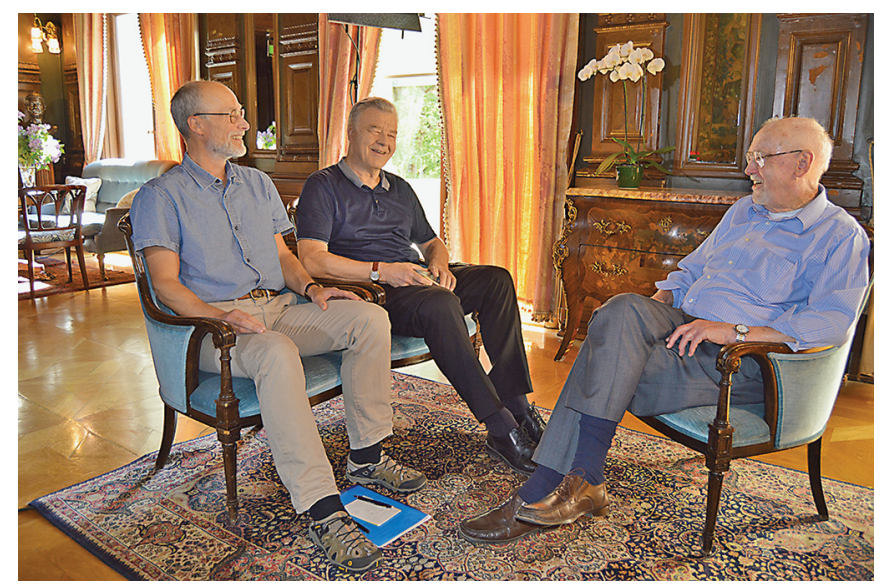

Figure 4. From left to right: Bjørn lan Dundas, Christian Skau, and Robert P. Langlands.

smaller. You look at the trace formula here and you look at the trace formula there, and they cancel. In other words, you come from one place and you look to see what it cancels-it cancels something-and you go along and along and along and you know you understand it. Ultimately, the real building blocks are those things in the big group that come from the trivial group. So, the last stage is to analyse those. I take the small group and I want to send it to the big group and I just have to look: I take the trace formula up here and it cancels everything I know from this. It just cancels everything; I said it should be made up by pieces and each should come from smaller groups and this just comes from the smaller group, and this comes from the smaller group, and this comes from the smaller group, and then I have to be careful because it can come from a bigger group and from a smaller group, and I have to be careful so I don't count it twice. So, I say they should be equal. I have to have a clear view of the combinatorics. Everything comes from a smaller group and some of it comes from two smaller groups and some is coming from three and so on. This depends on the image group. So, to show that this is really true, I just show that somehow the trace formula gives the same up here as it does for something in the selection of the various groups. This is pretty vague but in principle it is not so bad. And this is how it works but up until now at a very low level.

Dundas and Skau: So, that is at the forefront of your investigation?

Langlands: I mean, that is at the forefront of Arthur's investigation. I think if you want to hear what is available along these lines, you have to ask Arthur.

Dundas and Skau: We understand you are currently thinking in more differential geometric terms?

Langlands: I was thinking about the geometric theory and the geometric theory is not the trace formula, right? The geometric theory is basically Yang-Mills theory.

There are two papers-a brief one in English was premature and not entirely reliable. The other, which is longer and-so far as I know-reliable, is in Russian. This is already an obstacle but it is also very difficult to understand, in part because very few people, perhaps no one, understands the connection with Yang-Mills as in the paper of Atiyah-Bott. I might be able to help you with further questions but I have had difficulties with one Russian speaker who, in spite of encouragement, still does not understand the basic idea of the paper. He is a well-regarded mathematician. So it appears that the paper is difficult. I am nonetheless confident that it is correct. You might ask around!

Dundas and Skau: That is a recent paper of yours that we can read?

Langlands: It can be found on the web. ${ }^{4}$

Dundas and Skau: In 1872, Felix Klein launched his famous Erlangen programme. To every geometry he associated an underlying group of symmetries. Klein stated in his autobiography that the Erlangen programme remained the greatest guiding principle, or "leitmotiv," for his subsequent research. Do you see any analogy with the Langlands programme?

Langlands: I would hesitate to use the word programme but I think probably that leitmotiv is right. In other words, you have these two somewhat surprising structures on both sides: groups moving from one side to another. You have one side that is arithmetic and the other side that is analytic (or geometric, depending upon your view). So, you move around and you know that everything can go to $\mathrm{GL}_{n^{\prime}}$ and with $\mathrm{GL}_{n}$ you have this one example of an Euler-product that you can analytically continue. These things give you a very clear focus-or leitmotiv if you like-on what one should try to achieve.

Dundas and Skau: In 2016, we interviewed Andrew Wiles, who was awarded the Abel Prize for his proof of the modularity theorem for semistable elliptic curves, from which the Fermat theorem follows. The modularity theorem fits into the Langlands programme and Wiles expressed the sentiment that its central importance in mathematics lent him courage: one simply could not ignore it-it would have to be solved!

You propose a theory of mathematics that is rather encompassing: it is not a particular thing; it is a structural thing. What are your comments on this?

Langlands: I think what one is looking for is a structural thing. All of the particular instances are of interest. Or something like that. There is so much you just can't do that I hesitate to answer really. But, if you like, you have this one structure on the one side, the Diophantine equation, which is sort of embedded in one of the automorphic forms. Automorphic forms have a lot of intricate structure on their own, so you have a lot of information about the $L$-functions there that moves back here, i.e. to the Diophantine side, and that is usually what you want. But I am not a specialist in those things.

$\overline{4 \text { pub1ications.ias.edu/rp1/section } / 2659}$ 
Dundas and Skau: Both Harish-Chandra and Grothendieck-two mathematicians we know you admired-were engaged in constructing theories, not being satisfied with partial insights and partial solutions. Do you feel a strong affinity with their attitude?

Langlands: I greatly admired both of them and, incidentally, do not feel that I am at their level. Their impulses were, however, different. Grothendieck himself has described his own impulses. Harish-Chandra never did. He just went where the material led him. He abandoned the mathematics of his youth, as a student in India, on which he wrote many papers, and turned to the topic of his thesis with Dirac: representation theory, a theory that was gaining in popularity and depth when he came to the IAS with his advisor Dirac. He just went where it led him. In retrospect, he just went where his strength and ambition took him. Incidentally, his thesis was, in contrast to what followed, not very impressive.

Dundas and Skau: To what extent has it been important to you to be around people and in an environment where new ideas circulate?

Langlands: There were two people who made an absolute difference to my mathematical life. The first was Edward Nelson, whom I met basically by accident as a graduate student-I had come as a graduate student with a friend, who was an instructor at Yale, to the IAS to visit some of my friend's friends from his graduate-student days at Chicago, one of whom was Nelson. An incidental consequence of an informal conversation that day, during which we discussed mathematical matters of common interest, was that Nelson suggested to the Princeton mathematics department, where he was to begin teaching the following year, that I be offered a position as an instructor-no application, no documents, nothing.

The second is Salomon Bochner, who, after hearing me talk in an informal Princeton seminar, urged me to move from the rational number field to arbitrary number fields and to study the work of Hecke. He also recommended me to Selberg. As a consequence, I had my one and only mathematical conversation with Selberg. It was, of course, he who talked.

Harish-Chandra, too, made an enormous difference, principally because of his papers (these I read on my own initiative, many years before meeting him) but also because my appointment to the IAS was made-I suspect-at his initiative. I should also observe that it was a young Princeton colleague (although they were older than me) who directed me to Harish-Chandra's papers. So the answer to your question is certainly 'yes.' I owe a great deal to my education at UBC, where a very innocent young man, a boy if you like, was introduced to intellectual possibilities to which he has been attached all his life, and to Yale, where for two years he followed his own whims and where there were mathematicians who supported his independence. Whatever reservations I have about Princeton and its two academic establishments, it is clear from the preceding remarks that I am indebted in a serious way to specific individuals who were attached to them.

Dundas and Skau: Perhaps before we conclude the interview, it might be interesting to hear whether you have private, non-mathematical passions or interests of some sort, e.g. music, literature, language or poetry?

Langlands: Passions? I don't have any passions. But, you know, it is true that you want to take a look at other things, you know. History is fascinating: modern history, ancient history, the Earth's history, the Universe's history-these things are all fascinating. It is a shame to go through life and not have spent some time contemplating on that-certainly not everything of course but just to think about it a little bit.

Dundas and Skau: On behalf of the Norwegian Mathematical Society and the European Mathematical Society, and the two of us, we would like to thank you for this very interesting interview, and again congratulations on the Abel Prize.

Langlands: Thanks for inviting me.

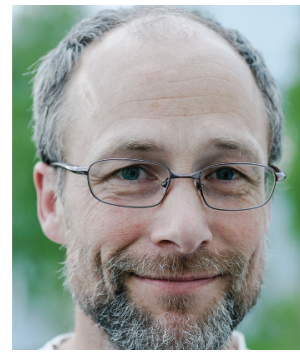

Bjørn Ian Dundas

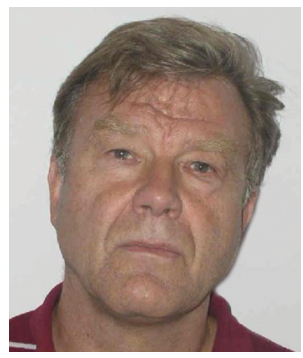

Christian Skau

\section{Credits}

Figure 1 is by Thomas Brun/NTB Production.

Figure 2 ๑Randall Hagadorn/Institute for Advanced Study.

Figure 3 ๑Institute for Advanced Study/Dan Komoda.

Figure $4{ }^{\circ}$ Anne-Marie Astad / The Norwegian Academy of Science and Letters.

Photo of Bjørn Ian Dundas is by Halvard Fausk. Photo of Christian Skau is courtesy of Christian Skau. 